\title{
Spinodal decomposition in multicomponent fluid mixtures: A molecular dynamics study
}

\section{Laradji, Mohamed; Mouritsen, Ole G.; Toxvaerd, Søren}

Published in:

Physical Review E. Statistical, Nonlinear, and Soft Matter Physics

Link to article, DOI:

10.1103/PhysRevE.53.3673

Publication date:

1996

Document Version

Publisher's PDF, also known as Version of record

Link back to DTU Orbit

Citation (APA):

Laradji, M., Mouritsen, O. G., \& Toxvaerd, S. (1996). Spinodal decomposition in multicomponent fluid mixtures: A molecular dynamics study. Physical Review E. Statistical, Nonlinear, and Soft Matter Physics, 53(4), 36733681. https://doi.org/10.1103/PhysRevE.53.3673

\section{General rights}

Copyright and moral rights for the publications made accessible in the public portal are retained by the authors and/or other copyright owners and it is a condition of accessing publications that users recognise and abide by the legal requirements associated with these rights.

- Users may download and print one copy of any publication from the public portal for the purpose of private study or research.

- You may not further distribute the material or use it for any profit-making activity or commercial gain

- You may freely distribute the URL identifying the publication in the public portal 


\title{
Spinodal decomposition in multicomponent fluid mixtures: A molecular dynamics study
}

\author{
Mohamed Laradji, ${ }^{1}$ Ole G. Mouritsen, ${ }^{2}$ and Søren Toxvaerd ${ }^{3}$ \\ ${ }^{1}$ Department of Physics, University of Toronto, Toronto, Ontario, Canada M5S $1 A 7$ \\ ${ }^{2}$ Department of Physical Chemistry, The Technical University of Denmark, Building 206, DK-2800 Lyngby, Denmark \\ ${ }^{3}$ Department of Chemistry, H. C. Ørsted Institute, University of Copenhagen, DK-2100 Copenhagen, Denmark
}

(Received 17 August 1995)

\begin{abstract}
We have investigated the effect of the number $p$ of components on the dynamics of phase separation in two-dimensional symmetric multicomponent fluids. In contrast to concentrated two-dimensional binary fluids, where the growth dynamics is controlled by the coupling of the velocity field to the order parameter, leading to large growth-exponent values, the dynamics in multicomponent fluids $(p=3,4)$ is found to follow a $t^{1 / 3}$ growth law, where $t$ is time, which we relate to a long-wavelength evaporation-condensation process. These findings, which are proposed to be consequences of the compact domain structure persisting in multicomponent fluids, imply that hydrodynamic modes do not affect the dynamics of the phase separation in these systems.
\end{abstract}

PACS number(s): 64.70.Ja, 64.60.Cn, 68.10.- m, 05.70.Ln

\section{INTRODUCTION}

The dynamics of first-order phase transitions, such as ordering or phase separation of binary mixtures, is a very interesting and important problem and has consequently received a lot of attention during the past three decades [1-3]. Whereas the early-time dynamics is well understood in terms of the Cahn-Hilliard-Cook linear theory [4-7], the late-time dynamics is a highly nonlinear and complicated process. The classical theory of Lifshitz and Slyozov [8], applicable to the phase-separation process in very dilute systems, i.e., in systems where the volume fractions of the two components are very different, predicts that the dynamics in the late-stage regime is characterized by a single time-dependent length scale, corresponding, for example, to the average droplet size $R(t)$, which grows in time as $t^{n}$ with the growth exponent being $n=1 / 3$. As a result, the system exhibits a self-similar behavior manifested by the scaling of the correlation function $G(\chi)=g(r, t)$ in terms of a dimensionless scaling variable $\chi=r / R(t)$. The specific value of the growth exponent in the Lifshitz-Slyozov (LS) theory is a consequence of a long-wavelength evaporationcondensation process that embodies a conservation law for the order parameter. In other words, the separation process is due to the evaporation of material from droplets with radii smaller than a certain time-dependent critical radius and condensation of material onto droplets with radii larger than the critical radius. Extensions of the LS analysis showed that the growth exponent is independent of spatial dimension [9]. Although the LS analysis can only be performed on very dilute systems, using simple arguments, Huse has been able to generalize the theory to the case where the domain structure is interconnected [10]. Moreover, a considerable amount of experiments and several types of simulations on twoand three-dimensional systems showed that the growth exponent in binary alloys is indeed independent of the system composition and spatial dimension [1]. Recently, Monte Carlo simulations on simple multistate Potts models have shown that the growth exponent in phase separating alloys is also independent of the number of components [11].

Binary fluids, on the other hand, behave differently. Several experiments performed on three-dimensional concentrated binary fluids have shown that the late-time growth exponent assumes a value $n=1$ [12-15]. Siggia [16] has shown analytically that the velocity field causes a necking-down instability of the tubular and interconnected domain structure, thereby leading to a large growth exponent value $n=1$. At very late times, inertial effects may become very important, leading to a crossover to a growth exponent $n=2 / 3 \quad$ [17].

In two dimensions, similar linear analyses predict an advection-controlled dynamics with a growth exponent $n=1 / 2$ [18], followed by inertia-controlled dynamics with a growth exponent $n=2 / 3$ [17]. This behavior has recently been observed in a molecular dynamics simulation by Velasco and Toxvaerd [19] and Langevin simulations $[20,21]$. When the compositions of the two fluid components are very different, droplets have compact spherical shapes. This implies that the mechanism corresponding to hydrodynamic transport of material along the interfaces is not effective since it does not contribute to an increase in the average size of the droplets. Therefore the dominant growth mechanism at late times is coalescence resulting from the Brownian motion of the droplets. This mechanism leads to growth exponents $n=1 / 3$ and $n=1 / 2$ in three and two dimensions, respectively. Consequently, in contrast to alloys, the dynamics of phase separation in fluid mixtures depends on both spatial dimension and volume fraction.

Our goal in this paper is to investigate the effect of the number $p$ of components on the dynamics of the phase 
separation in fluid mixtures. For this purpose, we consider symmetric ternary $(p=3)$ and quaternary $(p=4)$ mixtures in two dimensions and simulate them by means of molecular dynamics. This simulational technique has an advantage over the time-dependent Ginzburg-Landau simulations based on model $H$ [22] since in the former approach the quenches are performed directly into the fluid phase and thus the hydrodynamic modes arise naturally from the microscopic interactions between the molecules. A brief report of some of the results for the $p=3$ system has appeared in Ref. [23].

The present paper is organized as follows. In Sec. II we present the model and describe the numerical techniques used in our simulations. In Sec. III we present our results for the two types of fluid mixtures studied: ternary and quaternary fluids. Finally, we summarize and discuss our results in Sec. IV.

\section{MODEL AND NUMERICAL METHOD}

In our simulation model, we consider $N$ monoatomic molecules interacting via an extended two-body 12-6 Lennard-Jones potential that was introduced earlier by Velasco and Toxvaerd [19] and has been used in several subsequent simulations [23-25]. The Hamiltonian of the system is given by

$$
\begin{aligned}
\mathcal{H}\left(\mathbf{p}_{1}, \ldots, \mathbf{p}_{N} ; \mathbf{r}_{1}, \ldots,\right. & \left.\mathbf{r}_{N}\right) \\
= & \sum_{i=1}^{N} \frac{\mathbf{p}_{i}^{2}}{2 \mu_{i}}+4 \epsilon \sum_{i<j}^{N}\left[u\left(\alpha_{i}, \alpha_{j} ; r_{i j}\right)\right. \\
& \left.-u\left(\alpha_{i}, \alpha_{j} ; r_{c}\right)\right] \theta\left(r_{c}-r_{i j}\right)
\end{aligned}
$$

with

$$
u(\alpha, \beta ; r)=\left(\frac{\sigma}{r}\right)^{12}-\left(2 \delta_{\alpha \beta}-1\right)\left(\frac{\sigma}{r}\right)^{6},
$$

and $\theta(x)$ is the unit step function, which equals one when $x>0$ and is zero otherwise. This ensures that the interaction potential is truncated and then shifted at $r_{c}$. As usual the cutoff distance is given by $r_{c}=2.5 \sigma$. In Eq. (1), $\mathbf{r}_{i}$ is the position of a molecule $i, \mathbf{p}_{i}$ is its momentum, $\mu_{i}$ is its mass (we consider the case where all particles have the same mass $\mu$ ), and $r_{i j}=\left|\mathbf{r}_{i}-\mathbf{r}_{j}\right|$ is the distance separating two molecules $i$ and $j$. The species index $\alpha_{i}$ of a molecule $i$ assumes the values $1,2, \ldots, p$ corresponding to the labeling of the $p$ different species $A, B, \ldots, P$.

The specific form assumed by the interaction potential ensures that the force between two molecules of different species is purely repulsive whereas, for like species, it is the standard Lennard-Jones potential. This model exhibits both solid and fluid phases within the coexistence region between the different components. However, in the present study we shall consider only quenches into the fluid phase.

The $N$ molecules are confined in a two-dimensional, square-shaped box of linear size $L$ and subject to periodic boundary conditions. Three- and four-component mixtures with equal concentrations are considered, i.e.,
$\rho_{A}=\rho_{B}=\cdots=\frac{1}{p} \rho$, where $\rho$ is the average fluid density and $p=3$ or 4 for the ternary or the quaternary mixtures, respectively. Initially, the system is prepared at very low density ( $\rho=0.01$, which is well inside the homogeneous disordered phase) and then instantaneously quenched thermally by rescaling all spatial distances in the system so that the final equilibrium state corresponds to a coexistence between the $p$ fluid phases. Immediately after the quench, the temperature is kept constant.

For our simulation, we have adopted the Nosé-Hoover technique by which one integrates the Hamilton equations [26-29]

$$
\frac{d \mathbf{r}_{i}}{d t}=\frac{\mathbf{p}_{i}}{\mu}
$$

and

$$
\frac{d \mathbf{p}_{i}}{d t}=\mathbf{f}_{i}-\eta \mathbf{p}_{i}
$$

where $\mathbf{f}_{i}=-\partial \mathcal{H} / \partial \mathbf{r}_{i}$ is the force acting on molecule $i$, and $\eta$ is a friction parameter needed to control the temperature. $\eta$ varies in time with the total excess kinetic energy according to

$$
\frac{d \eta}{d t}=\left(\sum_{i} \frac{\mathbf{p}_{i}^{2}}{\mu}-g k_{B} T\right) /\left(g k_{B} T \tau_{\eta}^{2}\right),
$$

where $g=2 N-2$ is the total number of degrees of freedom in a two-dimensional system of $N$ monoatomic molecules [30], and $\tau_{\eta}$ is a characteristic thermostat relaxation time. Eqs. (3) and (4) are integrated using the leapfrog algorithm

$$
\mathbf{r}_{i}(t+\Delta t)=\mathbf{r}_{i}(t)+\frac{\Delta t}{\mu} \mathbf{p}_{i}\left(t+\frac{\Delta t}{2}\right)
$$

and

$$
\begin{aligned}
\mathbf{p}_{i}\left(t+\frac{\Delta t}{2}\right)= & \mathbf{p}_{i}\left(t-\frac{\Delta t}{2}\right)\left[\frac{1-\frac{\Delta t}{2} \eta(t)}{1+\frac{\Delta t}{2} \eta(t)}\right] \\
& +\frac{\Delta t \mathbf{f}_{i}(t)}{\mu\left[1+\frac{\Delta t}{2} \eta(t)\right]}
\end{aligned}
$$

where $\Delta t$ is the integration time step.

The motivation for choosing such an algorithm lies in its time-reversal symmetry that ensures the reversibility of the microscopic dynamics [29]. The integration time step was taken to be $\Delta t=0.005 \tau$, where the time scale is $\tau=\sqrt{\left(\mu \sigma^{2} / \epsilon\right)}$. It is very important to note that the integration time step is much smaller than the mean collision time, which can be calculated from the memory function and the appropriate Mori coefficients. For the particular case of $k_{B} T=\epsilon$ and $\rho \sigma^{2}=0.8$, the mean collision time is $0.05_{3} \tau$.

Following a quench, the system is relaxed during a time period of $2000 \tau$. The total time span of each run is therefore short, e.g., only a few nanoseconds in the case of a particle mass corresponding to $\mathrm{Kr}$. In order to ensure that this dynamics reaches the asymptotic late-time regime, the quench has to be sufficiently deep since the speed of the separation process is controlled by the ex- 
cess mixing energy. This may be difficult to control in a real experiment, but it is straightforward in our model simulation to provoke the phase separation to occur on a short-time scale by simply choosing a pair potential Eq. (1) with a strong repulsion between unlike particles. In order to maintain the correct hydrodynamic modes under these extreme conditions it is required that the force gradient for pairs of unlike species during a time step is smaller than the force gradient for particle collisions. This requirement is fulfilled in the present simulation.

The domain growth is probed through three different measures for the average domain size: The first one is defined as the inverse of the first moment of the structure factor

$$
R_{1}(t)=2 \pi \frac{\sum_{q=0}^{q=q_{c}} S(q, t)}{\sum_{q=0}^{q=q_{c}} q S(q, t)}
$$

where $q_{c}$ is a large wave-number cutoff taken to be $2 / \sigma$ [the value for $q_{c}$ is chosen so that the time-independent broad peak due to short-range liquid ordering is not included in the summation of Eq. (8)]. The second measure $R_{2}(t)$ is calculated from the first zero of the real-space correlation function, which is defined as

$$
g(r, t)=\frac{1}{p} \sum_{i=1}^{p} \frac{1}{\rho_{i}^{2}}\left\langle\delta \rho_{i}(\mathbf{r}, t) \delta \rho_{i}(0, t)\right\rangle .
$$

Due to the fact that the separating domains in twodimensional symmetric ternaries, quaternaries, etc., are compact, one can further use the domain-size distribution $P(A, t)$ to extract a third measure $R_{3}(t)$ :

$$
R_{3}(t)=\sqrt{\int P(A, t) A d A},
$$

where $A$ is the domain area. Averages are performed over 20 and 11 runs, with different initial conditions, for the three-component and the four-component systems, respectively.

\section{RESULTS}

We now present the results of our simulations [31]. First we display in Fig. 1 the time evolution of the real-space configurations for the $(p=3)$ - and $(p=4)$ component systems. Unlike symmetric binary fluids, the domain structure in fluids containing more than two components with equal concentrations is compact, similar to that in ternary alloys (see Ref. [11]). We notice for both values of $p$ that as time lapses, the domains coarsen as the phase separation progresses. In the present study, the simulations are not long enough to observe full phase separation.

In Fig. 2(a), the time evolution of the average domain size for the three-component system, as calculated from the three measures in Eqs. (8)-(10), is displayed in a double-logarithmic plot. We observe that the three measures are very consistent with each other and that the average domain size follows an algebraic growth law $R(t) \sim t^{n}$, with $n=0.309$ using $R_{1}, n=0.348$ using $R_{2}$, and $n_{3}=0.342$ using $R_{3}$. We have estimated the accuracy of this exponent to be around $5 \%$.

Similarly, in Fig. 2(b) we display the growth data for the four-component system. As in the three-component system, the average domain size consistently grows algebraically in time with a growth exponent $n=0.333$ as calculated from $R_{1}, n=0.354$ as calculated from $R_{2}$, and $n=0.353$ as calculated from $R_{3}$.

The consistency in the values of the growth exponent and the finding of a value close to $n=1 / 3$ is an indication that the growth mechanism in both the threeand the four-component fluid mixtures is most probably the evaporation-condensation process responsible for the Lifshitz-Slyosov growth in Ostwald ripening [8]. We shall later argue that other mechanisms are unlikely to be operative.

In phase separation by Ostwald ripening, the total volume $\mathcal{V}$ of the ordering component is constant in time due to the conservation law. Therefore $\mathcal{N}(t)[R(t)]^{d} \sim \mathcal{V} \sim$ const, where $\mathcal{N}(t)$ is the total number of the growing droplets, $R(t)$ is the average domain size, and $d$ is the spatial dimension. Thus the total number of domains diminishes in time as $t^{-d n}$. We have calculated the time dependence of the average number of droplets in both the three- and the four-component systems and found that $\mathcal{N}(t) \sim t^{-2 / 3}$; cf. Fig. 3 , which is consistent with our predictions.

We now discuss why other mechanisms found in binary fluids cannot be operative in symmetric multicomponent fluids. Due to the compact structure of the growing domains in these systems, the hydrodynamic modes will try to make the domains more rounded, but this does not contribute to their coarsening. Moreover, Brownian diffusion of domains leading to coalescence, as observed in binary fluids with low volume fractions, cannot be operative in symmetric multicomponent fluids due to the fact that a given domain in our case is not surrounded by a single other domain, but rather by a number of different domains corresponding to different species. This drastically impedes the Brownian motion of the individual domains in the domain agglomerate and thus limits the coalescence process. We are aware that coalescence is still present in symmetric multicomponent fluids, but it is also present in multicomponent or dilute binary alloys in which hydrodynamic modes are absent. We therefore believe that the domains in symmetric multicomponent fluids are growing due to the long-range evaporationcondensation process.

The late-time growth is dominated by a single timedependent length scale, which scales all other length scales in the system. An obvious length scale in the system is the average domain size or the average distance between two neighboring domains. As a result, the structure factor can be written in a scaled form independent of time:

$$
F(x)=\frac{1}{[R(t)]^{d}} S(q, t),
$$




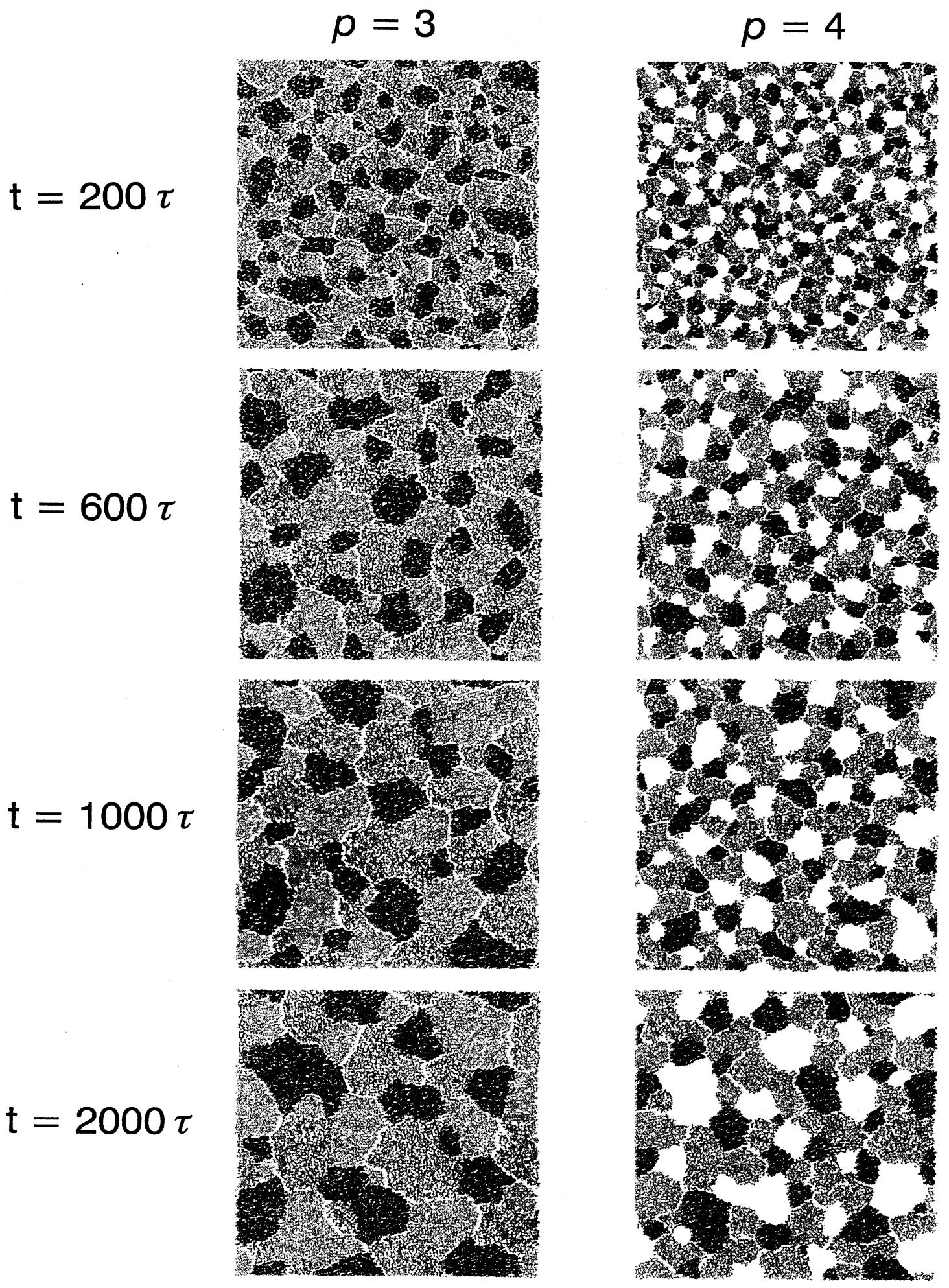

FIG. 1. Snapshots of the domain structure as a function of time $t$ formed during the phase-separation process in threecomponent $(p=3)$ and four-component $(p=4)$ symmetric mixtures. Different colors correspond to different species. 
where $x=q R(t)$ is a scaled wave number. By calculating the inverse Fourier transform of $S(q, t)$, one can show that the correlation function also can be written in a scaled form

$$
G(\chi)=g(r, t)
$$

where $\chi=r / R(t)$. In Figs. 4(a) and 4(b) the scaled structure factor in Eq. (10) is shown for the three- and the four-component systems, respectively. Scaling is observed to set in from about $200 \tau$, signaling that the systems we are dealing with are well within the asymptotic scaling regime. From the insets of Fig. 4, we notice that the structure factor starts to increase again at very large wave numbers. This increase is in fact part of the short- range liquid ordering peak, whose position and height are time independent, and therefore this peak does not comply with scaling. In Fig. 5 the scaled correlation functions [cf. Eq. (11)] are also found to exhibit an excellent scaling.

The investigation of the short-wavelength behavior of the structure factor shows that it follows

$$
S(q, t) \sim q^{-\alpha}
$$

as demonstrated in the insets of Figs. 4(a) and 4(b). This behavior is known as Porod's law and is the result of the scattering from the thin interfaces that separate the growing domains. For thin interfaces, one can show that the Porod exponent is $\alpha=d+1$. In the present case,
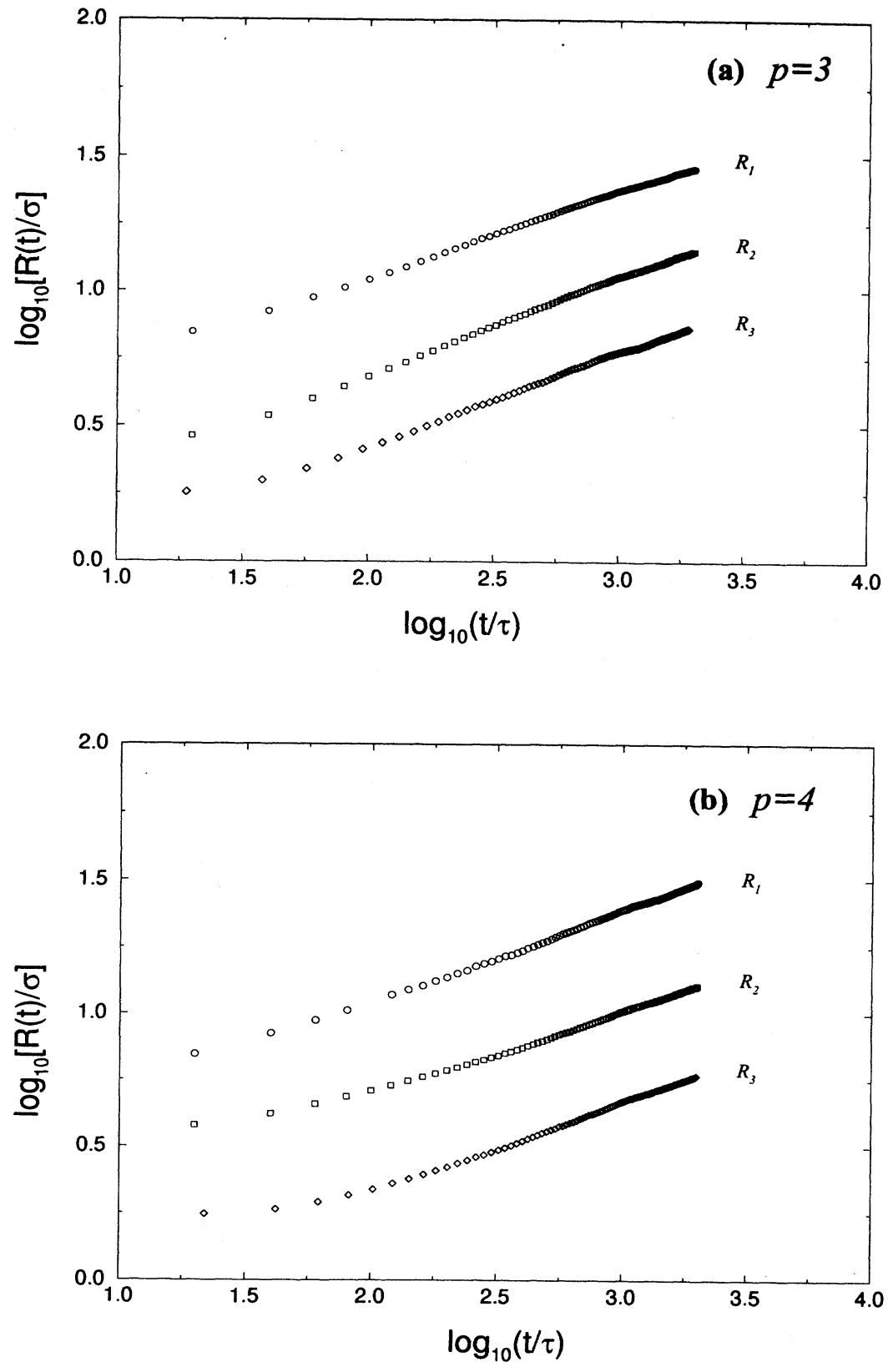

FIG. 2. Log-log plots vs time $t$ for the different measures of time-dependent length scale of the phase-separation process in (a) three-component $(p=3)$ and (b) fourcomponent $(p=4)$ symmetric mixtures. $R_{1}(t)$ is obtained from the first moment of the structure factor; cf. Eq. (3). $R_{2}(t)$ is the first zero of the correlation function. $R_{3}(t)$ is is the average linear domain size obtained from the domain-size distribution function; cf. Eq. (4). 

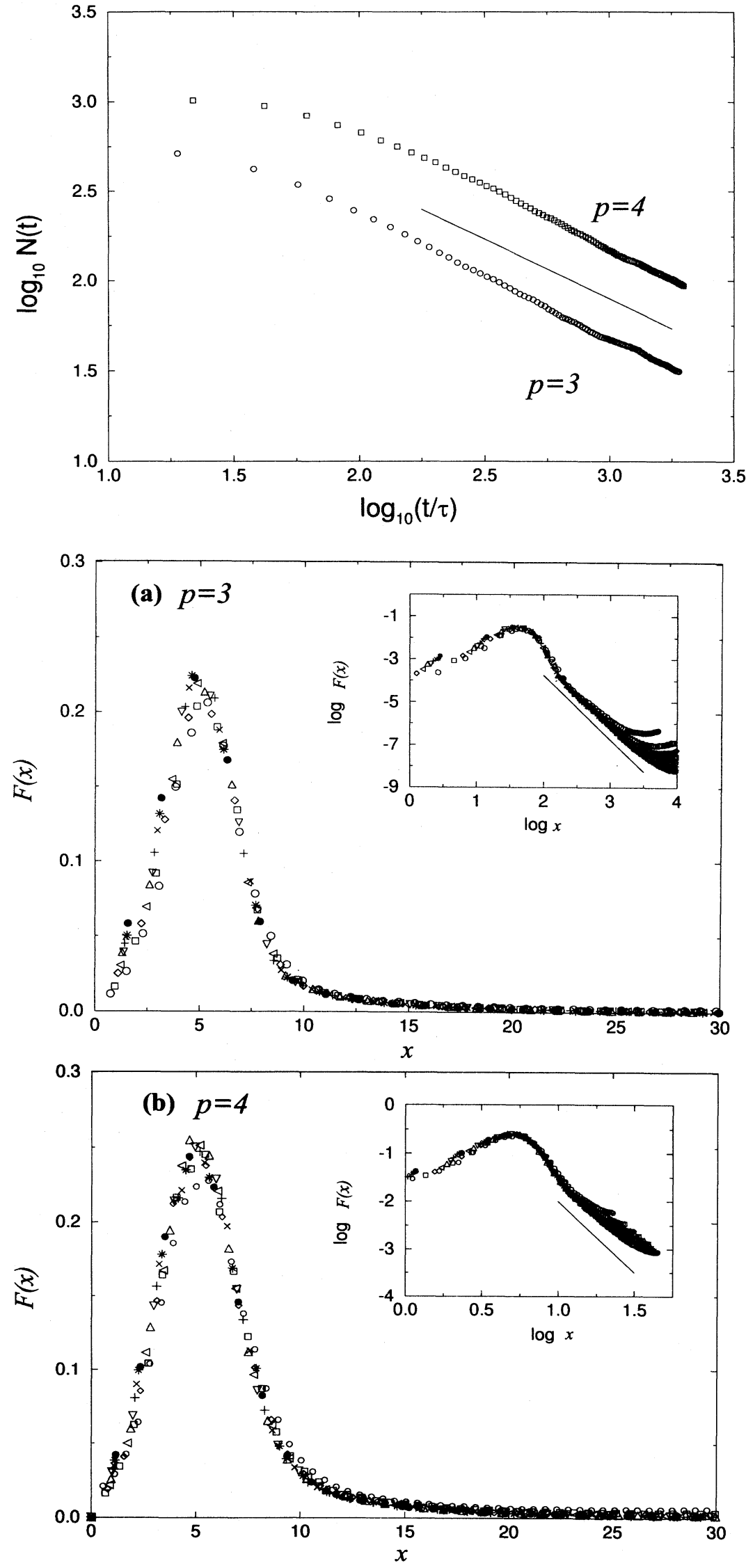

FIG. 3. Log-log plot of the average number $\mathcal{N}(t)$ of droplets as a function of time for three-component $(p=3)$ ( $(\circ)$ and fourcomponent $(p=4)(\square)$ symmetric mixtures. The slope of the solid line is $-2 / 3$.
FIG. 4. Scaled structure factor, $F(x, t)=$ $\left[R_{1}(t)\right]^{-2} S(q, t)$ with $x=q R_{1}(t)$, shown for (a) three-component $(p=3)$ and (b) four-component $(p=4)$ symmetric mixtures. The times shown are from $t=200 \tau$ up to $t=2000 \tau$ in steps of $200 \tau$ corresponding to the symbols in the sequence $\circ, \square, \diamond, \triangleleft, \triangle, \nabla,+, \times, *, \bullet$. The insets show $F(x, t)$ in a log-log plot. The solid lines have a slope of $\delta=-3$. 
we find that $\alpha=2.84$ for the three-component system and $\alpha=2.74$ for the four-component system. Since the interfaces (see Fig. 1) are not very rough, the presence of the vortex defects between three domains of different species may be responsible for the fact that the value of the Porod exponent in the case of two-dimensional multicomponent mixtures is slightly lower than $\alpha=3$.

\section{SUMMARY AND CONCLUSION}

In this paper we have studied in detail the dynamics of two-dimensional symmetric multicomponent fluid mixtures by means of molecular dynamics simulations. We considered both three-component and four-component mixtures. Our main result is that the growth exponent in both of these systems is $n=1 / 3$, as determined from several different length-scale measures. We believe that the specific growth law observed in our simulations is due to the long-wavelength evaporation-condensation process [8]. The growth law in these systems is therefore different from that in two-dimensional symmetric binary fluids where the growth exponent is given by $n=1 / 2$ for intermediate times crossing over to $n=2 / 3$ at later times. Nevertheless, these two growth-exponent values are consequences of the convoluted domain structure in binary mixtures, whereas in symmetric multicomponent systems the domains have a compact structure. However, binary fluids with low volume fractions that do display a compact domain structure exhibit a domain growth with an exponent $n=1 / 2$ due to coalescence resulting from the Brownian motion of the domains. The compact domain structure in multicomponent fluids suppresses this Brownian motion and therefore an $n=1 / 2$ growth exponent is not observed.

Recently, Chen and Lookman [32] have investigated the dynamics of phase separation in two-dimensional symmetric multicomponent fluids and dilute binary fluids by means of lattice-Boltzmann simulations and found
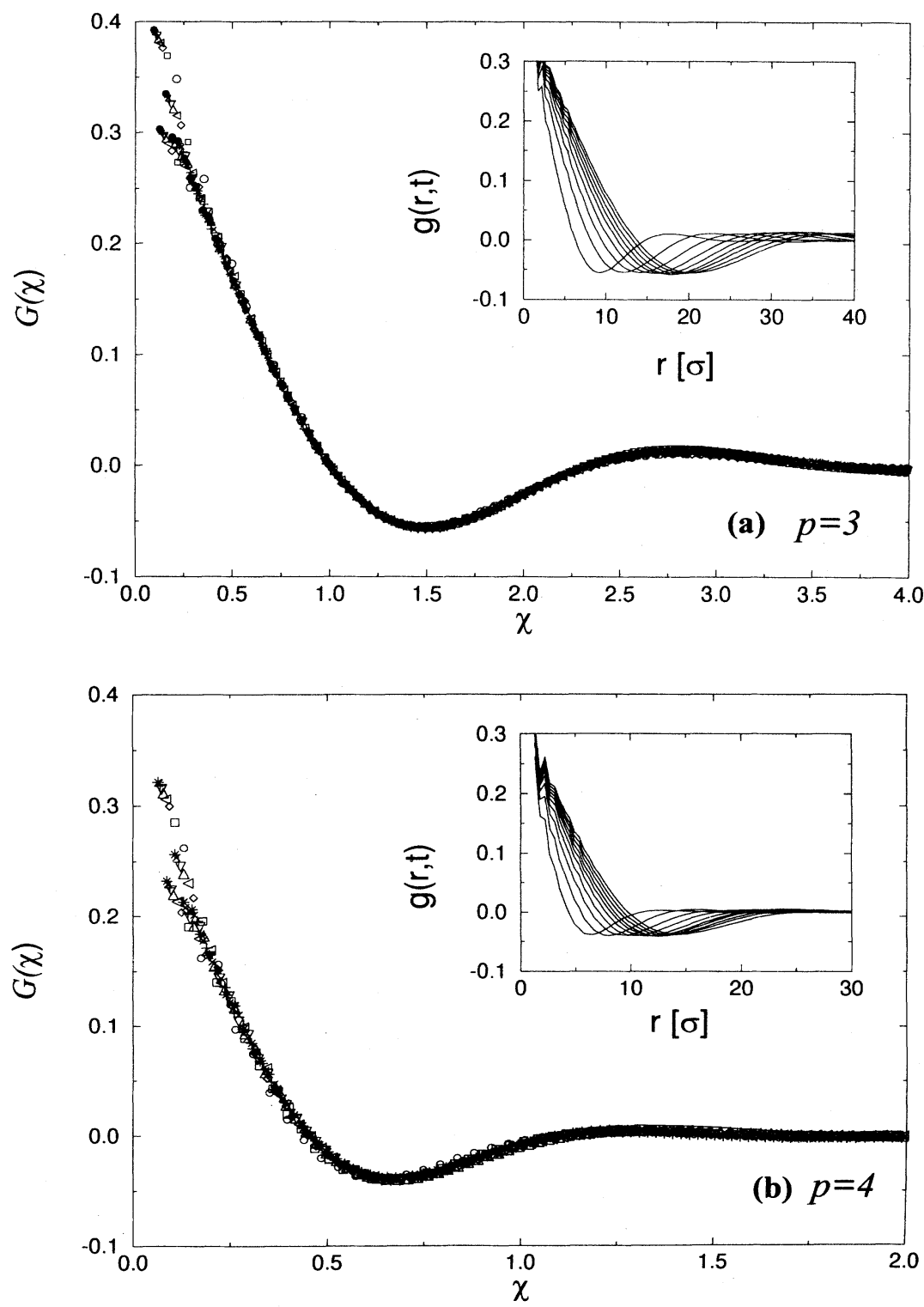

FIG. 5. Scaled real-space correlation function $G(\chi)=g(r, t)$ with $\chi=r / R(t)$ for (a) a three-component fluid mixture $(p=3)$ and (b) a four-component fluid mixture $(p=$ 4). The symbols are the same as those used in Fig. 4. The times shown are from $t=200 \tau$ up to $t=2000 \tau$ in steps of $200 \tau$. The insets show the time evolution of $g(r, t)$ from $t=200 \tau$ up to $t=200 \tau$ in steps of $200 \tau$. 
results that conflict with ours. In particular, they found, for both symmetric multicomponent fluids and dilute binary fluids, an intermediate-time regime during which domains are convoluted and grow as $t^{1 / 3}$ followed by a faster growth regime with an exponent $n=2 / 5$ characterized by a compact domain structure. They associate the later regime to the hydrodynamic effects and explained it in terms of a dimensional analysis due to Furukawa for dilute binary fluids [17]. But their latetime growth exponent for the dilute binary fluids is different from $n=1 / 2$, as discussed above. In fact, a recent Langevin simulation at off-critically quenched fluids by Wu et al. [20] finds an exponent that is close to $1 / 2$. We are unable to resolve the discrepancy between our results and theirs. Of course, we cannot exclude the possibility that another growth regime may prevail at very late times. However, a study of much larger systems at much longer times is required in order to resolve this question, which, unfortunately, at the moment is prohibitive due to insufficient computer power.

We have also investigated the late-time behavior of the dynamics and found dynamical scaling in the structure factor as well as the correlation function for both threeand four-component mixtures, indicating that our investigation is safely within an asymptotic late-time regime. Our conclusion is therefore that the dynamics in fluid mixtures does not only depend on the spatial dimension and volume fraction but also on the number of components and their composition. Hence the phase-separation dynamics in fluid mixtures is more complex and therefore richer than that in alloys due to the different competing mechanisms.

As a consequence of the results presented in this paper, several interesting questions arise. For example, how does a ternary fluid mixture phase separate in the case where the concentration of one of the components is much smaller than the two others? If the concentrations of the two majority components are comparable, the domains will be interconnected, and the dynamics of these mixtures may therefore be effectively similar to that of symmetric binary fluids. Another interesting question relates to a symmetric multicomponent fluid in three dimensions. Due to the higher spatial dimensionality of this system, the domains do not have to be compact but can be convoluted $(p \leq d)$. Hence the domain growth may be similar to that in dense binary fluids, i.e., leading to a growth exponent $n=1$ at intermediate times followed by $n=2 / 3$ at very late times. Such a study would therefore be of substantial interest and is therefore currently in progress in our laboratory.

\section{ACKNOWLEDGMENTS}

This work was supported by the Danish Natural Science Research Council and the Danish Technical Research Council. The stay of M.L. in Denmark was supported by the Danish Natural Science Research Council under Grant No. 11-0474-1.
[1] J.D. Gunton, M. San Miguel, and P.S. Sahni, in Phase Transitions and Critical Phenomena, edited by C. Domb and J. L. Lebowitz (Academic, New York, 1983), Vol. 8, p. 265.

[2] Dynamics of Ordering Processes in Condensed Matter, edited by S. Komura and H. Furukawa (Plenum, New York, 1987), p. 574.

[3] O.G. Mouritsen, in Kinetics of Ordering and Growth at Surfaces, edited by M. G. Lagally (Plenum, New York, 1990), p. 1.

[4] J.W. Cahn and J.E. Hilliard, J. Chem. Phys. 31, 688 (1959).

[5] H.E. Cook, Acta Metall. 18, 297 (1970).

[6] K.R. Elder, T.M. Rogers, and R.C. Desai, Phys. Rev. B 38, 4725 (1988).

[7] M. Laradji, M. Grant, M.J. Zuckermann, and W. Klein, Phys. Rev. B 41, 4646 (1990).

[8] I.L. Lifshitz and V.V. Slyozov, J. Phys. Chem. Solids 19, 35 (1962).

[9] J.H. Yao, K.R. Elder, H. Guo, and M. Grant, Phys. Rev. B 45, 8173 (1992); 47, 14110 (1993).

[10] D.A. Huse, Phys. Rev. B 34, 7845 (1986).

[11] C. Jeppesen and O.G. Mouritsen, Phys. Rev. B 47, 14724 (1993).

[12] N.-C. Wong and C.M. Knobler, Phys. Rev. A 24, 3205 (1981).

[13] P. Guenoun, R. Gastaud, F. Perrot, and D. Beysens,
Phys. Rev. A 36, 4876 (1987).

[14] F.S. Bates and P. Wiltzius, J. Chem. Phys. 91, 3258 (1989).

[15] T. Izumitani, M. Takenaka, and T. Hashimoto, J. Chem. Phys. 92, 3213 (1990).

[16] E.D. Siggia, Phys. Rev. A 20, 595 (1979).

[17] H. Furukawa, Adv. Phys. 34, 703 (1985).

[18] M. San Miguel, M. Grant, and J.D. Gunton, Phys. Rev. A 31, 1001 (1985).

[19] E. Velasco and S. Toxvaerd, Phys. Rev. Lett. 71, 388 (1993); Phys. Rev. E (to be published).

[20] Y. Wu, F.J. Alexander, T. Lookman, and S. Chen, Phys. Rev. Lett. 74, 3852 (1995).

[21] T. Lookman, Y. Wu, F.J. Alexander, and S. Chen (unpublished).

[22] Hohenberg and Halperin have classified critical dynamics in binary mixtures into dynamical models. Model $B$, for instance, describes the dynamics in a binary mixture with conserved order parameter. See P.C. Hohenberg and B.I. Halperin, Rev. Mod. Phys. 49, 435 (1977).

[23] M. Laradji, O.G. Mouritsen, and S. Toxvaerd, Europhys. Lett. 28, 157 (1994).

[24] M. Laradji, O.G. Mouritsen, S. Toxvaerd, and M.J. Zuckermann, Phys. Rev. E 50, 1243 (1994).

[25] G. Leptoukh, B. Strickland, and C. Roland, Phys. Rev. Lett. 74, 3636 (1995).

[26] S. Nosé, Mol. Phys. 52, 255 (1984). 
[27] W.G. Hoover, Phys. Rev. A 31, 1695 (1985).

[28] S. Toxvaerd, Mol. Phys. 72, 159 (1991).

[29] S. Toxvaerd, Phys. Rev. E 47, 343 (1993).

[30] Notice that since the momentum is conserved, the total number of degrees of freedom should be $d N-d$ instead of $d N$, where $d$ is the spatial dimension.

[31] Some of these results were briefly presented in Ref. [23].

[32] S. Chen and T. Lookman, J. Stat. Phys. 81, 223 (1995). 


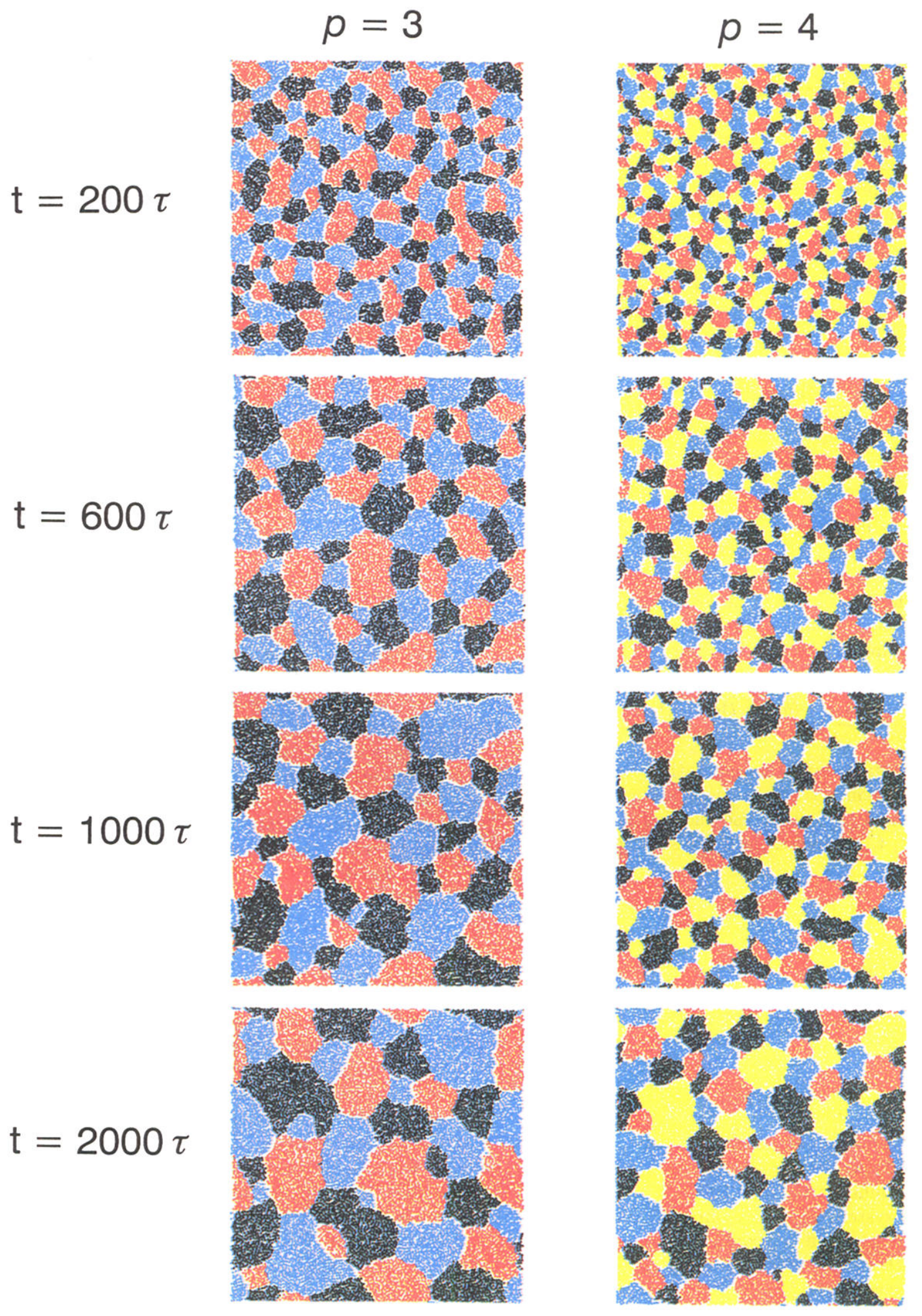

FIG. 1. Snapshots of the domain structure as a function of time $t$ formed during the phase-separation process in threecomponent $(p=3)$ and four-component $(p=4)$ symmetric mixtures. Different colors correspond to different species. 